\title{
Wavefield Simulation in General Elastic and Viscoelastic Fractured Media Based upon the Pseudo-spectral Method
}

\author{
Ik Bum Kang ${ }^{1}$ and How-Wei Chen ${ }^{2, *}$
}

(Manuscript received 27 May 2003, in final form 20 October 2003)

\begin{abstract}
This study demonstrates synthesis of seismic responses in general elastic and viscoelastic fractured media using a two-dimensional pseudo-spectral method. From the full waveform simulation approach, different types of source configurations with corresponding wavefields and synthetic records are generated. Viscoelastic wavefield responses, which incorporate quality factors for both $P$-waves $(Q p)$ and $S$-waves (Qs) through the superposition of relaxation mechanisms and velocities for attenuative and dispersive media, are presented, tested and implemented to assess their potential for practical application. The modeling results show that the more time that elapses, the less the wave amplitudes can be observed in a viscoelastic media than in elastic media. The simulation of both pressure and shear source fully illustrate the corresponding decomposed waves and their corresponding seismograms. Also, the synthetic model of the anticline structure embedded in the layered model shows that diffraction responses are directly related to the fracture/fault zone structure. From the prescribed model, transmission, reflection, and converted multi-phase events can readily be identified in both elastic and viscoelastic media. Aside from this, evaluating seismic responses using a specially designed source and receiver configuration, such as horizontal drilling technique, is critical in studies pertaining to reservoirs. It is also demonstrated that by examining such seismic responses, the effects of fracture distributions and the corresponding time-lapsed monitoring of oil/gas leakage within a reservoir can be evaluated in advance by the proposed method for numerical studies.
\end{abstract}

(Key words: Viscoelastic, Wave propagation, Numerical simulation, Pseudo-spectral method, Fracture media, Source radiation pattern, Reservoir modeling and monitoring)

\footnotetext{
${ }^{1}$ Korea Institute of Geosciences \& Mineral Resources, Korea

${ }^{2}$ Institute of Seismology \& Institute of Applied Geophysics, National Chung Cheng University, Taiwan

${ }^{*}$ Corresponding author address: Prof. How-Wei Chen, Institute of Seismology \& Institute of Applied Geophysics, National Chung Cheng University, Min-Hsiung, Chia Yi, 621, Taiwan, ROC; E-mail: seiswei@eq.ccu.edu.tw
} 


\section{INTRODUCTION}

Conventional elastic forward modeling typically employs $\mathrm{P}$ - and S-wave velocities as well as density for the simulation of a full seismic wavefield. However, certain well-known phenomena - i.e., the attenuation effects caused by earth heterogeneities and physical rock processes - are usually not taken into account because of the difficulty in quantitatively analyzing the attenuation effects. With the help of current high-performance computing capabilities and discrete theory, the use of the relaxation mechanism has made the numerical simulation approach much more feasible and efficient when it comes to simulating the attenuation effects through the parameterization of the quality factors $(\mathrm{Q})$. Such attenuation phenomena can be attributed to grain boundary relaxation, thermoelasticity, diffusional motion of dislocations, point defects, or among other factors. The attenuation effects are, of course, directly related to the well-known, corresponding quality factors parameters for P-waves (Qp) and S-waves (Qs), together with the Qp/Qs ratio, since both parameters are closely related not only to sediments but also to tectonic conditions (Dougherty and Stephen 1988; Clouser and Langston 1991). Studies of the phenomena of scattering, intrinsic and coda $\mathrm{Q}^{-1}$ base on observation (Phillips et al. 1988; Mayeda et al. 1992; Fehler et al. 1992); origin of coda waves (Aki and Chouet 1975); basic mechanism and models (Frankel and Wennerberg 1987; Toksoz et al. 1988); the relationship between spectral decay parameter $\mathrm{k}$, attenuation time $\mathrm{t}^{*}$ and attenuation effect on source spectrum (Anderson and Hough 1984) all play important roles in seismology studies.

In this study, a simulation of the more realistic seismic responses using the quality factors for $\mathrm{P}$ - and S-waves, as well as velocity and density is undertaken following the viscoelasticity theory and numerical pseudo-spectral method presented in the following sections. Description of the major equations involve in viscoelastic wave propagation theory are presented as a basis for revealing its numerical implementation. Various aspects in computational efficiency, accuracy requirements and numerical implementation procedures, characterization of source signature and propagation effects from fractured media are emphasized and explicitly discussed. Intrinsic attenuation and scattering from the effects of a fractured media or the undulation of structure boundary are explicitly considered for wave phenomena studies.

\section{VISCOELASTIC WAVE PROPAGATION THEORY}

For the simulation of viscoelastic wave propagation, Carcione et al.'s (1988a, b, and c) formulation for viscoelasticity was used to define quality factors that can be implemented with the superposition of relaxation mechanisms through relaxation times. The equations of motion in a 2-D isotropic linear viscoelastic medium (Carcione 1988a, b, c, 1990) are:

$$
\rho U_{t t}=\left[\left(\lambda_{u}+2 \mu_{u}\right) U_{x}+\lambda_{u} V_{z}+\frac{1}{2} \sum_{l=1}^{L_{1}} e_{l l}+\sum_{l=1}^{L_{2}} e_{11 l}\right]_{x}+\left[\mu_{u}\left(U_{z}+V_{x}\right)+\sum_{l=1}^{L_{2}} e_{12 l}\right],
$$

and 


$$
\rho V_{t t}=\left[\lambda_{u} U_{x}+\left(\lambda_{u}+2 \mu_{u}\right) V_{z}+\frac{1}{2} \sum_{l=1}^{L_{1}} e_{1 l}+\sum_{l=1}^{L_{2}} e_{11 l}\right]_{z}+\left[\mu_{u}\left(U_{z}+V_{x}\right)+\sum_{l=1}^{L_{2}} e_{12 l}\right]
$$

where $U$ and $V$ are the horizontal and vertical displacements; $x$ and $z$ are the horizontal and vertical space coordinates; $t$ is time; $\rho$ is density; $L$, and $L_{2}$ are the numbers of dilatation and shear relaxation mechanism present (usually $L_{l}$ and $L_{2}=2$ are sufficient); subscripts $x$, $z$, and $t$ denote partial differentiation of the subscripted quantity with respect to the subscript variable; $e_{1 l}, e_{11 l}$, and $e_{12 l}$ are the memory variables; $\lambda_{u}$ and $\mu_{u}$ are the un-relaxed Lamé parameters (defined below).

Let $M_{u 1}$ and $M_{u 2}$ be the un-relaxed (viscoelastic) dilatation and shear moduli; $M_{1}$ and $M_{2}$ be the relaxed (pure elastic) dilatation and shear moduli; and $\tau_{\sigma l}^{(v)}$ and $\tau_{e l}^{(v)}$ be the stress and strain relaxation times. Then,

$$
\begin{aligned}
& M_{u \nu}=M_{v}\left[1-\sum_{l=1}^{L_{v}}\left(1-\frac{\tau_{e l}^{(v)}}{\tau_{\sigma l}^{(v)}}\right)\right], \quad \text { for } v=1,2, \\
& \lambda_{u}=\frac{1}{2}\left(M_{u 1}-M_{u 2}\right),
\end{aligned}
$$

and

$$
\mu_{u}=\frac{M_{u 2}}{2} .
$$

Finally, the memory variables are

$$
\begin{gathered}
\dot{e}_{1 l}=-\frac{e_{1 l}}{\tau_{\sigma l}^{(1)}}+\phi_{1 l}\left[U_{x}+V_{z}\right], \\
\dot{e}_{11 l}=-\frac{e_{11 l}}{\tau_{\sigma l}^{(2)}}+\phi_{2 l}\left[U_{x}+V_{z}\right],
\end{gathered}
$$

and

$$
\dot{e}_{12 l}=-\frac{e_{12 l}}{\tau_{\sigma l}^{(2)}}+\frac{1}{2} \phi_{2 l}\left[U_{z}+V_{x}\right],
$$

where the $\cdot$ denotes a time derivative, $\phi_{1 l}$ and $\phi_{2 l}$ are the relaxation functions

$$
\phi_{v l}=\frac{M_{v}}{\tau_{\sigma}^{(v)}}\left(1-\frac{\tau_{e l}^{(v)}}{\tau_{\sigma l}^{(v)}}\right), \quad \text { for } v=1,2
$$

The important theoretical background and wave equations fully describe the fundamental principles involved in vecoelastic wavefield simulation. In this case, the elastic wave propagation theory becomes a special case. 


\section{THE NUMERICAL PSEUDO-SPECTRAL METHOD}

A number of recurring problems in seismology, such as locating earthquake, lateral changes in the Earth material in terms of isotropic, anisotropic or inelastic properties and source, path and site amplification effects are of great important. Modeling the velocity and density structure of the earth with heterogeneity can not simply be represented as a system of layered media. A clear-cut analytic solution to these problems is not simple; thus, some type of perturbation or numerical solution is necessary. The finite-difference (FD) and the finite-element (FE) methods are considered typical numerical solutions to the problems of simulating wave propagation and have, therefore, been continuously studied since the first FD paper was published (Alterman and Karal 1968).

When a high-frequency response is desired, an alternative is to employ the pseudo-spectral (PS) method, whereby spatial calculations of derivatives are performed by multiplication in the Fourier domain (Kreiss and Oliger 1972; Gazdag 1973; Orzag 1981; Holberg 1987). In light of the advantages of having fast convergent rate, which enables stable computations, along with its high degree of accuracy in describing attenuation and dispersion behavior, this method is employed successfully in this study to simulate viscoelastic wave propagation responses within an entire computational grid.

To attain the same degree of accuracy, in fact, the numerical PS method requires as few as a quarter of the number of grid points for the fourth-order FD, and one-sixteenth of the number of points for the second-order FD approximation (Fornberg 1987). Since the first PS method for use in seismological studies was proposed by Kosloff and Baysal (1982), the numerical method not only has been applied to 2-D forward seismic modeling (Chen and McMechan 1992a, b; Kang and McMechan 1990; Kang and McMechan 1993) and 3-D simulations (Kosloff 1990; Chen and McMechan 1993; Reshef et al. 1988) but also has been used for prestack reverse-time depth migration (Chen and McMechan 1992c). Slightly later, Chen (1996) presented a method to improve viscoacoustic wavefield simulation using the staggered grid PS technique which emphasizes its numerical characteristics to reduce oscillating signals from coarse grid computations. Based on these previous efforts, it is understandable that simulating a quasi-linear vector quantity of viscoelastic responses in which the phenomena of amplitude decay and waveform dispersion under certain specific initial and boundary conditions has become one of the key issues among seismologists over the past ten years.

The computational procedures for viscoelastic wavefield simulation are as follows:

1) Given the (real) relaxed moduli $M_{1}$ and $M_{2}$, which may be computed from input elastic compressional and shear velocities and density, and the stress $\left(\tau_{\sigma l}\right)$ and $\operatorname{strain}\left(\tau_{e l}\right)$ relaxation times, we can compute the complex moduli $M_{v}^{c}(\omega)$ (Carcione 1988b).

$$
M_{\nu}^{c}(\omega)=M_{\nu}\left[1-L_{v}+\sum_{l=1}^{L_{v}} \frac{1+i \omega \tau_{e l}^{(v)}}{1+i \omega \tau_{\sigma l}^{(v)}}\right], \quad \text { for } \mathrm{v}=1,2
$$

The relaxation times are chosen to provide the desired behaviors of the quality factors $Q_{p}$ and $\mathrm{Q}_{\mathrm{s}}$ (for $\mathrm{P}$ and $\mathrm{S}$ waves respectively) as functions of frequency $(w)$, 


$$
Q_{p}(\omega)=\frac{\operatorname{Re}\left(M_{1}^{c}+M_{2}^{c}\right)}{\operatorname{Im}\left(M_{1}^{c}+M_{2}^{c}\right)},
$$

and

$$
Q_{s}(\omega)=\frac{\operatorname{Re}\left(M_{2}^{c}\right)}{\operatorname{Im}\left(M_{2}^{c}\right)} .
$$

From equations (6), (7), and (8), it is clear that a combination of relaxation times that produce any given $Q(\omega)$ is not unique; this implies that many different physical causes of viscoelasticity in terms of the total number of mechanisms involved may behave similarly.

2) The un-relaxed Lamé parameters $\lambda_{u}$ and $\mu_{u}$ are computed from the un-relaxed moduli using equations ( 3 ).

3) The equations of motion (1) are solved by leap-frog time stepping approach. At each time step, a new value of each of the three memory variables is obtained by solving equations (4) and is updated to the previous total wavefields by the sum of (1) over the numbers of dilatation and shear relaxation mechanisms.

Generating synthetic seismograms in two-dimensional media is achieved by solving equations (1) through (8). Numerical models are parameterized by Lamé parameters $(\lambda, \mu)$, density $(\rho)$ and qualify factors $\left(Q_{p}\right.$ and $\left.Q_{s}\right)$ specified on a regular two-dimensional grid. Initial values of stresses, and the first time derivatives of stresses, are set to zero for the first-time step. The horizontal and vertical components of displacement, $U$ and $V$, can be derived by the explicit numerical finite-difference calculation in the time domain,

$$
U_{t t}^{n}=\left(\frac{\partial}{\partial t} U^{n}-\frac{\partial}{\partial t} U^{n-1}\right) / \Delta t
$$

and

$$
V_{t t}^{n}=\left(\frac{\partial}{\partial t} V^{n}-\frac{\partial}{\partial t} V^{n-1}\right) / \Delta t,
$$

where, $n$ and $n-1$ refer to the present and the previous time steps respectively, and $\Delta t$ is the time-sampling increment. Initial values in equation (9) are set to zero.

The distribution of source function in both space and time is defined by spatial differentiation of the Gaussian scalar potential (Kosloff et al. 1984),

$$
X(t, x, z)=\exp \left[-\alpha\left(t-t_{0}\right)^{2}\right] \exp -\left[\left(x-x_{0}\right)+\left(z-z_{0}\right)\right]^{2},
$$

where $t$ is time, $\alpha$ defines the width of the wavelet, and $x_{0}$ and $z_{0}$ are horizontal and depth position of the source. $t_{0}$ is a time shift required to make the wavelet causal. Pressure and shear sources can be inserted separately and combined in arbitrary proportions. Thus, a variety 
of wave events can be easily distinguished from each other by comparing the results from modeling with pre-defined pressure and shear sources.

Numerically-induced dispersion can result from aliasing in spatial derivative calculations and/or from inaccuracies in time derivative calculations. Kosloff and Baysal (1982) pointed out, by phase velocity calculations, the stability condition is

$$
V_{p} \Delta t / \Delta x \leq 0.2,
$$

where $V_{p}$ is the P-wave velocity, and $\Delta x$ the grid increment in the $x$ direction must be satisfied. Base on our test, negligible dispersion occurs even for the Nyquist limit of two grid points per wavelength through iterative time marching scheme. Thus, in the case of forward seismic modeling as a means to simulating wave propagation in seismic prospecting and for pure earthquake studies, stable numerical calculations are obtained by satisfying the precondition of maintaining stability, as set forth in equation (11). In the examples presented here, free surface boundary and absorbing boundary conditions are applied on the corresponding top and at the edges of the computation domain in such a way that no artificial waves can be reflected. Hence, non-physical waves can be sufficiently suppressed when absorbing boundary condition is applied.

The 2-D modeling algorithm described above enables a synthesis of the seismic responses for an extensive variety of realistic cases in both exploratory and seismological studies. In the following synthetic examples, all of the models are defined by their viscoelastic properties and attenuation factors. The simulation of the wave propagation phenomena can be computed and visualized via a series of fixed-time snapshots through the model domain or directly from the theoretical calculations of synthetic responses recorded at each pre-defined station location (seismograms) in the space-time domain. The computed synthetic seismograms and wavefield snapshots do not show the absorbing areas surrounding the numerical model. For simplicity, the Q-boundaries defined in the models are assumed to be the same as the velocity boundaries.

\section{SIMULATION OF SOURCE RADIATION AND MODE CONVERSION AT A FREE SURFACE}

Study of the source signatures is essential in computing synthetic seismograms in that entire wavefield responses are directly related to the specified source radiation pattern (Chen and McMechan 1992a). The straightforward way to evaluate qualitatively the behavior of a point source signature is to observe the resulting wavefronts as a snapshot shortly after the excitation of the source. The fundamental difference in the source radiation pattern is illustrated by using a simple homogeneous model (Fig. 1 a) where a buried line source (in 2-D) is initiated at the desired location. The model is composed of isotropic homogeneous elastic and viscoelastic properties with specified parameters for the P-and S-wave velocities, density, and quality factors.

For general two-dimensional media, it is important to consider the characteristic of source signature in full seismic wavefield studies. For the numerical calculations in the source signa- 
ture study, a grid size of $0.02 \mathrm{~km}$, a time sampling of 0.001 seconds, and a dominant frequency of $50 \mathrm{~Hz}$ are used. For the purposes of simplicity, only elastic responses are used to demonstrate different types of source radiation patterns. Figure 2 depicts the four basic point-source configurations that are implemented in our PS computations, namely pure pressure, pure shear, double couple source (dip angle $=0$.) and directional horizontal force source signature. The decomposed horizontal (Fig. 2a) and vertical (Fig. 2b) source wavefield signatures are discernable and essential when the identification of an individual event is required in the full waveform simulation and for the interpretation of reflections induced by the structure (Chen and McMechan 1992b).

In wave propagation studies and in the related applications in seismic prospecting, separation of P- and S- displacement wavefields can be easily obtained through basic mathematical curl and divergence operators (Olsen and Schuster 1995). For real world situation, such a processing procedure may not be practical enough as field data may suffer from coherent seismic noise, diffractions and multiples. A more straightforward way is to simulate pure explosive (scalar field, Fig. 2, first panel) and pure shear (vector field, Fig. 2, second panel) sources simultaneously under parallel computing environments using MPI (Message Passing Interface) or PVM (Parallel Virtual Machine). Thus, taking advantage of current parallel computing technique has its unique way of solving practical problem. Earthquake studies use doublecouple source signatures (Fig. 2, third panel) for numerical green function computation, in which both pressure and shear waves are generated to investigate wave propagation in complex subsurface structures for studying path and site effects. Despite the generation of pure explosive and shear sources, the implementation of a directional force is essential in simultaneously generating P- and S-waves (Fig. 2, last panel), and here, it is treated as a single couple source signature. Linear combination of individual directional forces with a specified radiation angle, in fact, produces a more complicated source signature. From Fig. 2, energy partition and the corresponding radiation pattern are different, they thus can serve as a basis for interpreting synthetic and field records, as shown in the following sections.

Although the source radiation pattern can be simulated numerically, a thorough descrip-
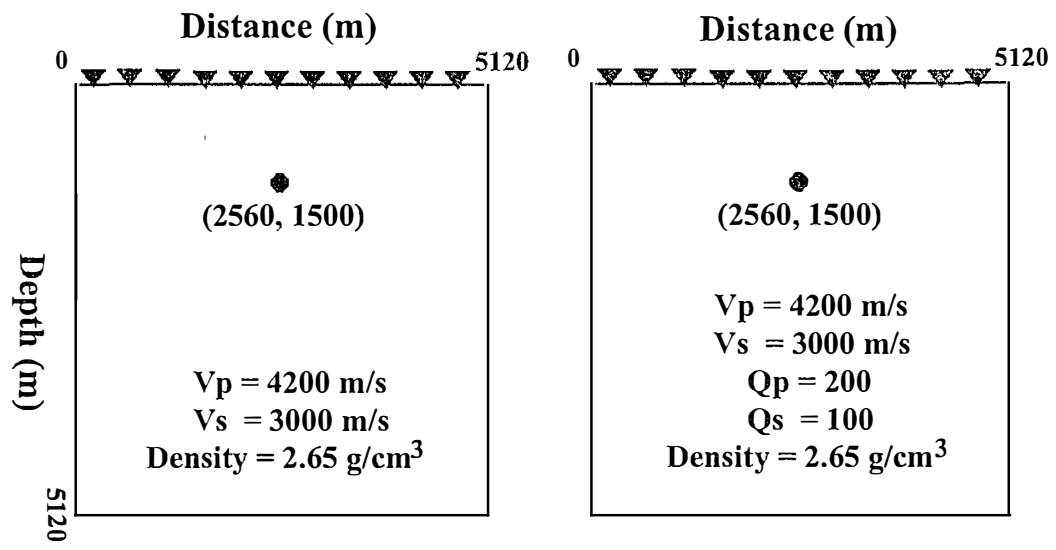

Fig. 1. Sources and their corresponding receiver array in homogeneous elastic (left) and viscoelastic (right) model with specified medium parameters. 
(a)
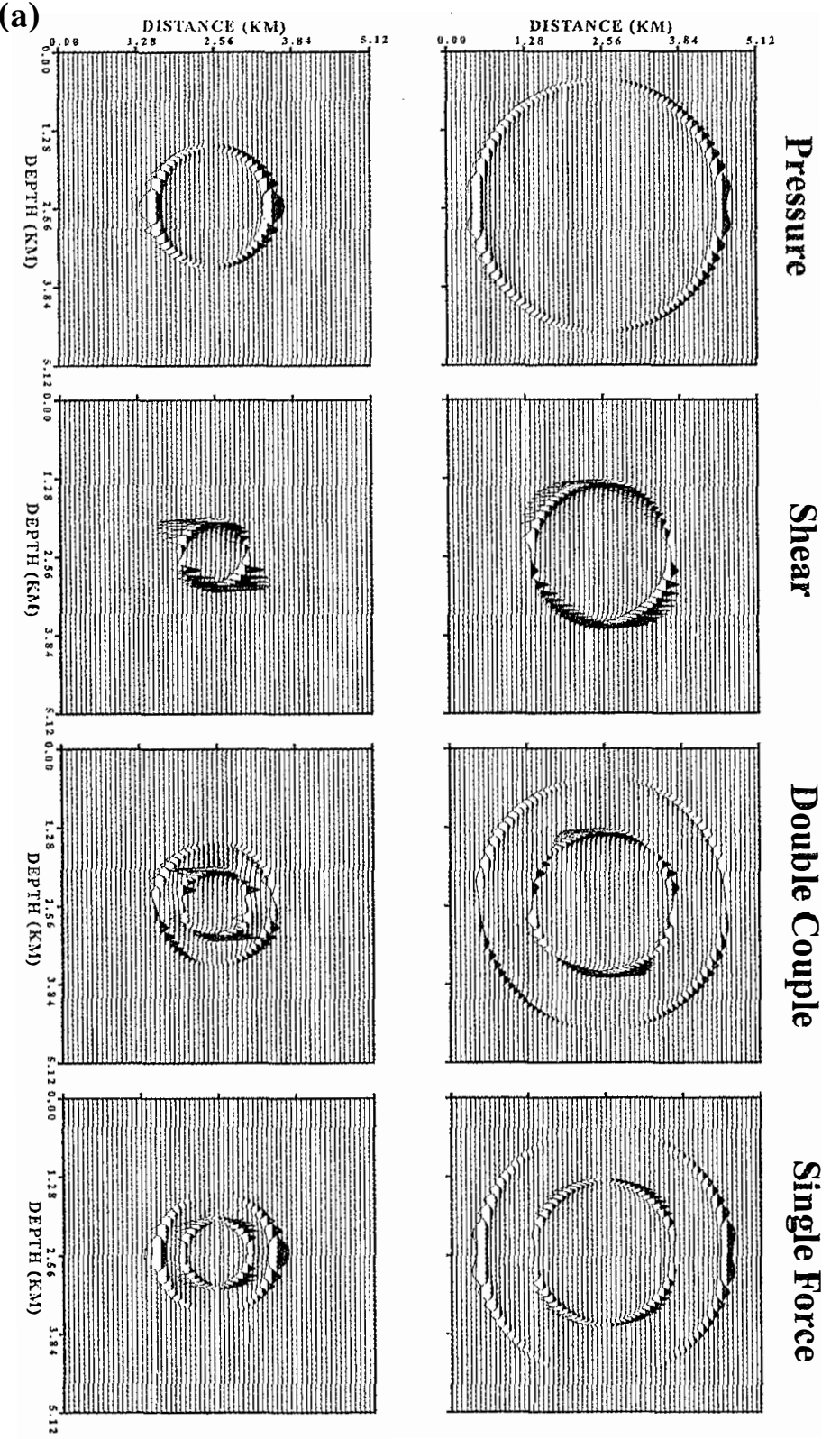

苟

Fig. 2. (a) Computation of full elastic waveform responses related to four basic types of source signatures. Snapshots at 0.25 second (left) and $0.50 \mathrm{sec}-$ ond (right) are shown for horizontal component source wave field generated by the PS method. (b) Computation of full elastic waveform responses related to four basic types of source signatures. Snapshots at 0 . 25 second (left) and 0.50 second (right) are shown for vertical component source wave field generated by the PS method. 
(b)
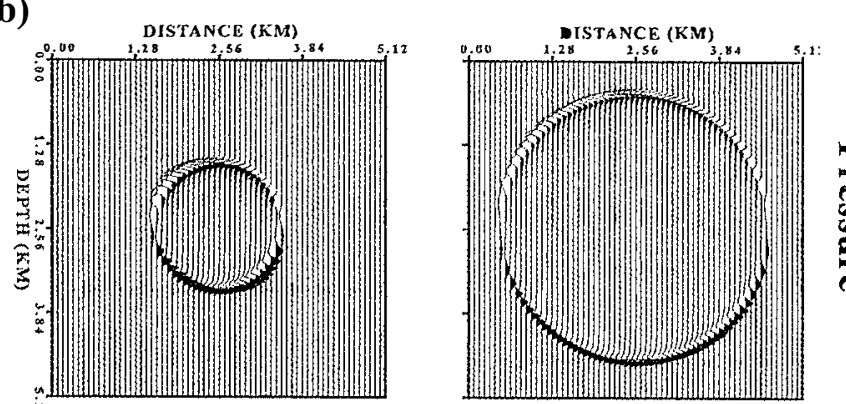

0
0
0
0
0
0
0
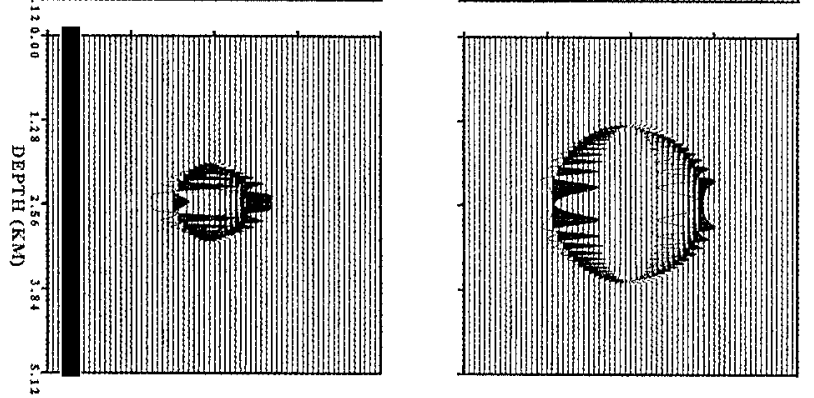

\section{$\frac{6}{8}$}
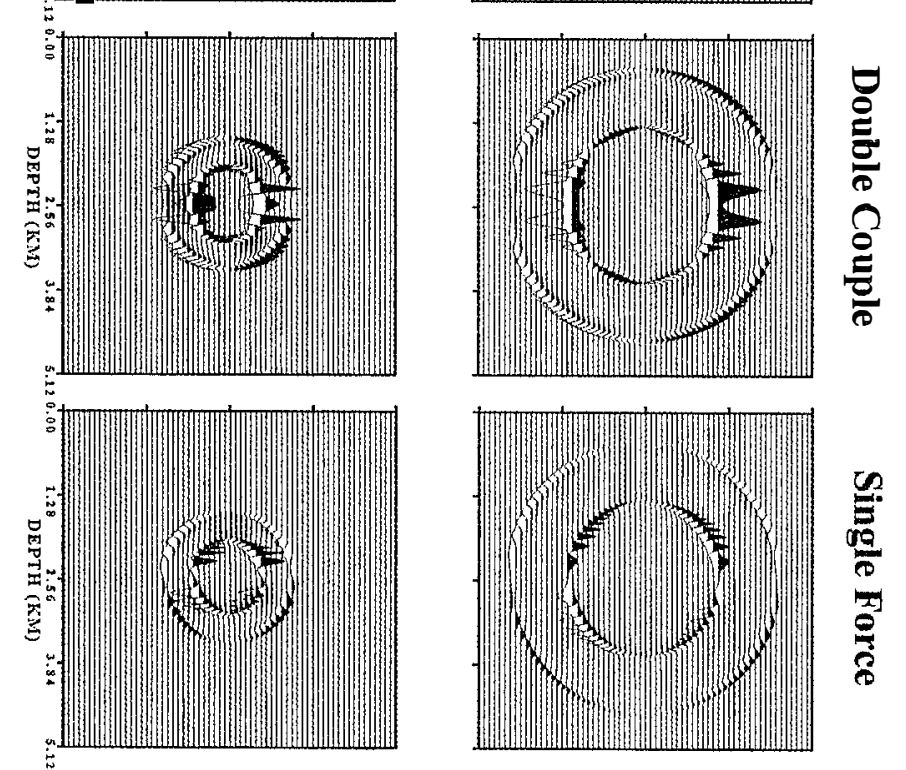

(Fig. 2. Continued)

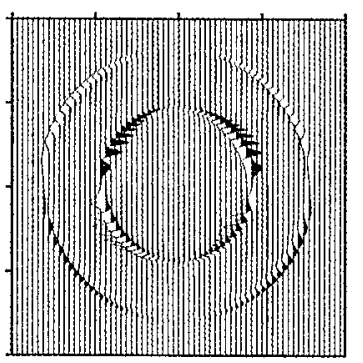

告

tion of wave generation caused by conversion at the free-surface is another important issue which makes entire wavefield computations slightly complicated. To assess their accuracy, the free-surface boundary conditions (Grant and West 1965) are that the normal and the tangential stresses are all zero:

$$
\lambda \frac{\partial U}{\partial x}+(\lambda+2 \mu) \frac{\partial V}{\partial z}=0,
$$


(a)
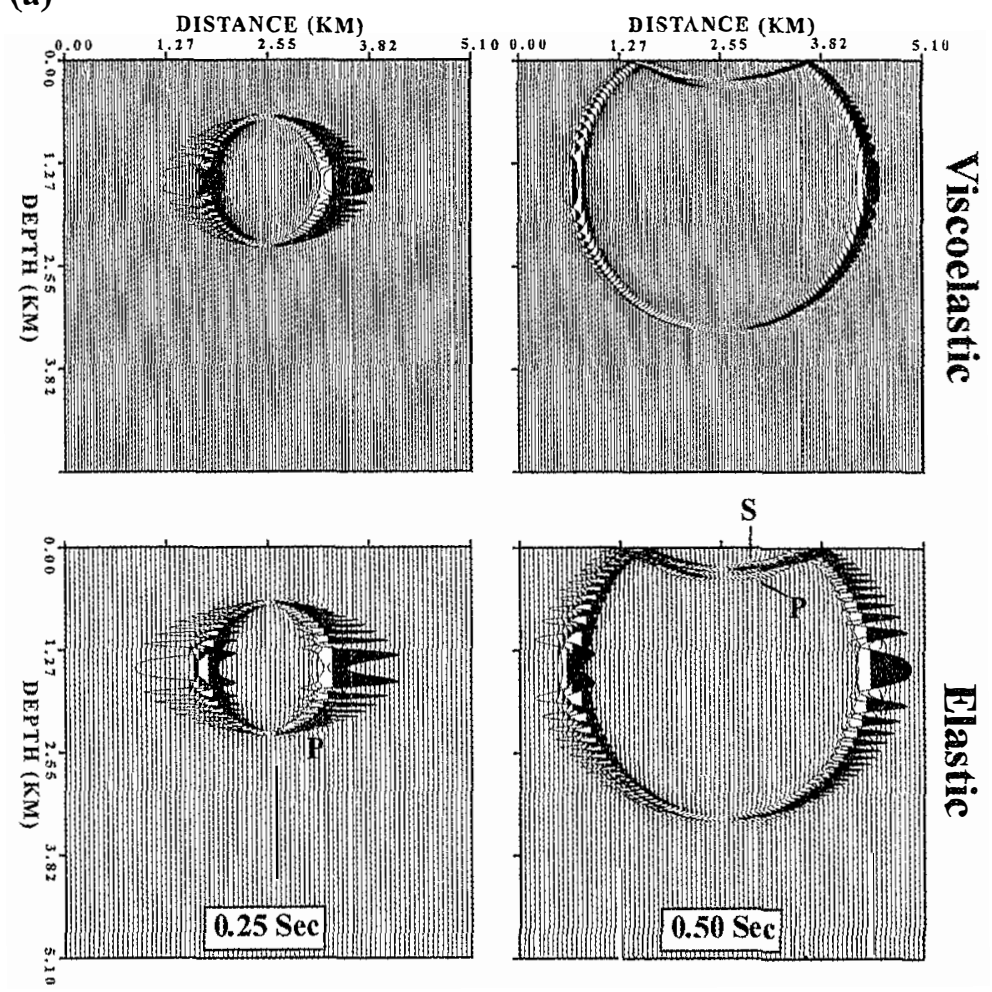

Fig. 3. (a) Mode conversion occurred at free surface from a compression source. The accuracy of pseudo-spectral computation can be achieved by solving boundary conditions in equation 12 . Horizontal component of P-to-P and P-to- $\mathrm{S}_{\mathrm{v}}$ waves are visible to show their fundamental responses in both elastic and viscoelastic media. Amplitude decay in viscoelastic media becomes quiet substantial. (b) Mode conversion occurred at free surface from a compression source. The accuracy of pseudo-spectral computation can be achieved by solving boundary conditions in equation 12 . Vertical component of P-to-P and P-to- $\mathrm{S}_{\mathrm{v}}$ waves are visible to show their fundamental responses in both elastic and viscoelastic media. Amplitude decay in viscoelastic media becomes quiet substantial.

and

$$
\frac{\partial U}{\partial z}+\frac{\partial V}{\partial x}=0 .
$$

These equations are implemented for all of the examples in this paper. Mode conversions between P-and Sv- wave at the free surface for a specified point-source signature in a homogeneous medium are presented to show the accuracy of the proposed method. In addition, the effects of intrinsic attenuation are also illustrated because of their simplicity and their general 
(b)

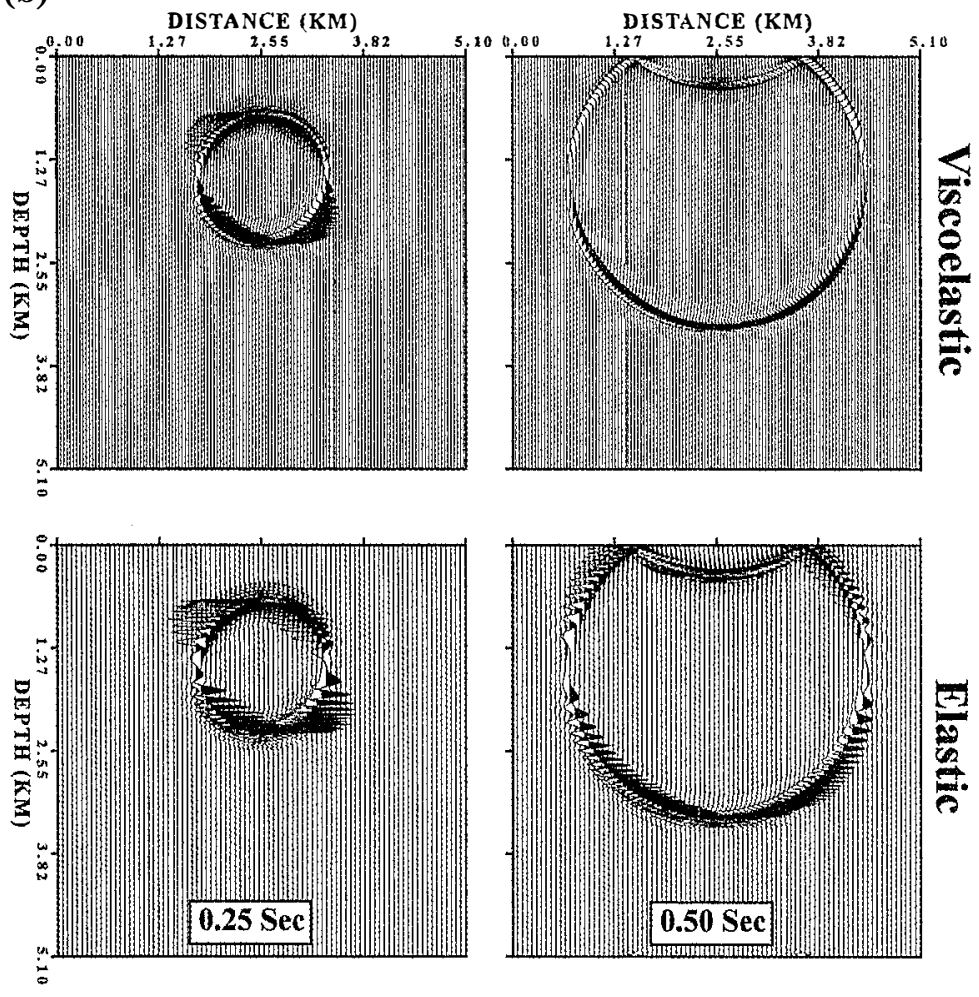

(Fig. 3. Continued)

propagation features in a homogeneous medium. Figures. 3a, b respectively show the time evolution of the vertical and horizontal displacements generated by a pure explosive source in the depth profile. In each figure, the panel on the left shows that the waves are generated soon after the source is excited at a representative time $(0.25 \mathrm{sec})$ before the wavefront from the primary source hits the free-surface. The panels on the right in Figs. 3a, b show the subsequent vertical and horizontal displacements. Noticeable S-wave converted from the free-surface can be observed, thus clearly demonstrating that the conventional simulation of acoustic waves is much too simplified to be used for conversion phase identification and interpretation. Albeit not the focus of our simple model study, for comparison, the attenuated waves are presented in the same format in Figs. 3a, b (upper panels) to show the variations in amplitude decay computed from the equation of motion in a viscoelastic medium compared with those in an elastic one, as shown in the lower panels.

Synthetic vertical and horizontal component seismograms can be produced for any desired recording point in the computational grid by extracting the vertical and horizontal amplitudes at that point for a sequence of time steps, and then visualizing these values as a time series. For a line of recorders along the surface, as shown in Fig. 1, the horizontal components of both elastic and viscoelastic responses are displayed and they are compared in Fig. 4 for a point of pure explosive source. Figure 5 shows the corresponding horizontal component seis- 


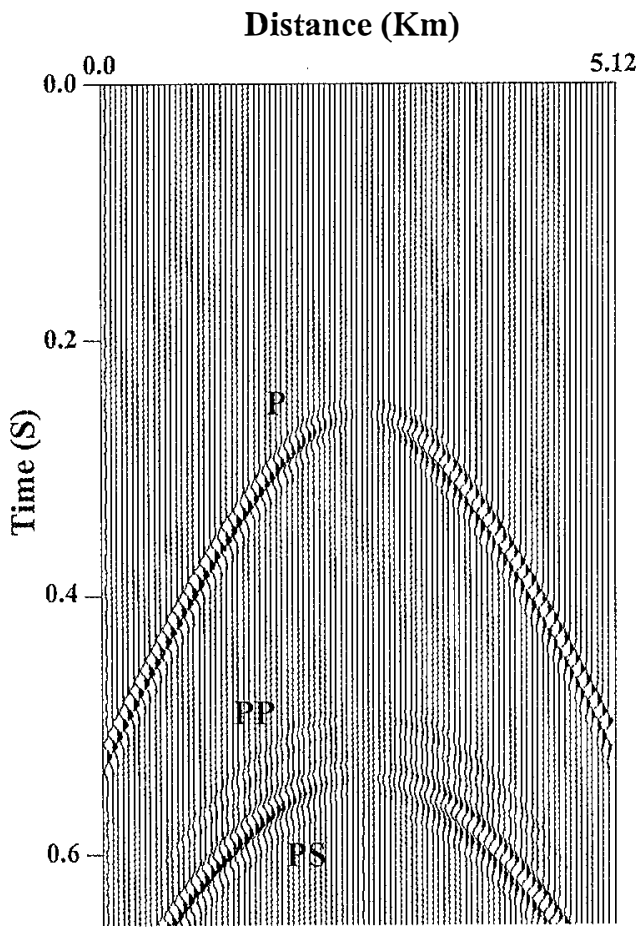

Elastic

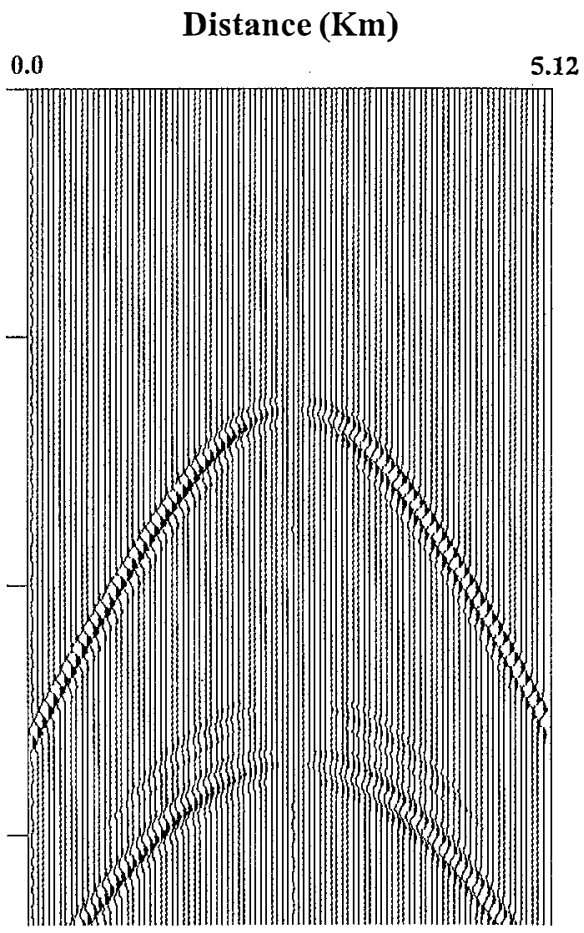

Viscoelastic

Fig. 4. The horizontal component of synthetic displacement seismograms derived from a pure explosive (pressure) source in the models of Fig. 1.

mograms generated by a point source with pure P- and S-sources excited simultaneously. Keeping the source signature in mind, it is relatively easy to identify the P- and S- waves, as seen in Figs. 4 and 5. The conversion phases generated at a free-surface can be distinguished by the significant differences in their amplitude level. In addition, the differences in the moveout curve of the P- and S-arrivals are mainly determined by the $\mathrm{P}$ - and $\mathrm{S}$-wave propagation velocities. The attenuation of amplitudes in the seismic records from a viscoelastic response (with quality factors), unlike that of the amplitudes which decays solely due to geometrical spreading in an elastic medium, is attributed to the cumulative effects as the propagation distance increases. Upon examination of the seismic responses from synthetic snapshots and seismograms, it is apparent that the amplitude response of later arrivals in the viscoelastic medium is further attenuated than that in the elastic medium.

\section{WAVE PROPAGATION IN FRACTURED MEDIA}

Seismic wave propagation in oil, gas and water reservoir environments has become an important research topic in reservoir engineering and environmental studies, especially when enhancement in oil/gas recovery (EOR) or in recovering water resource (EWR) issues are 


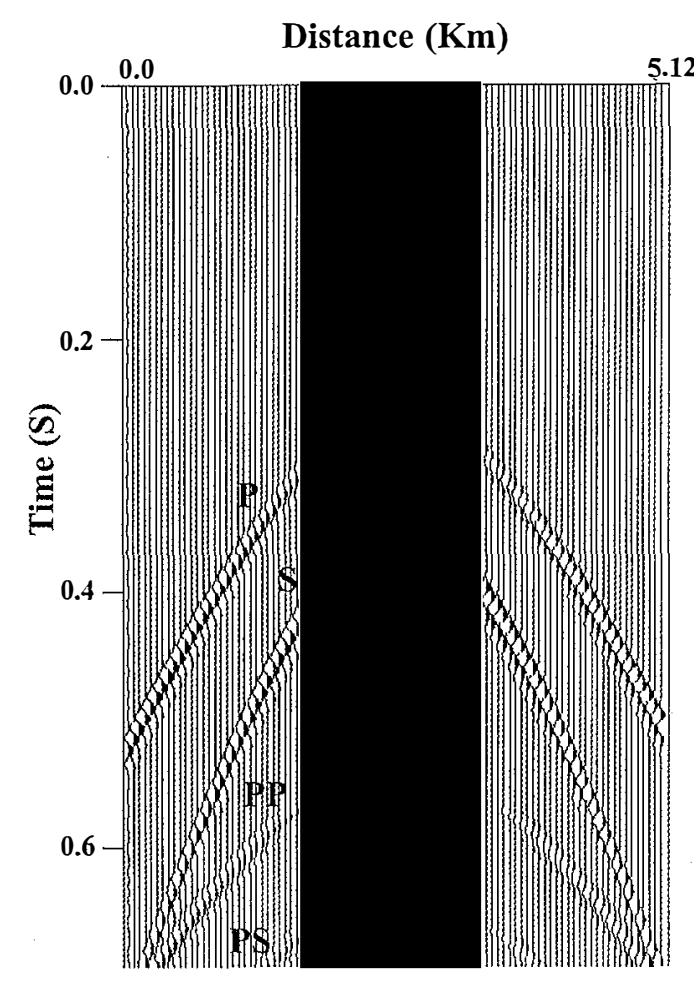

Elastic

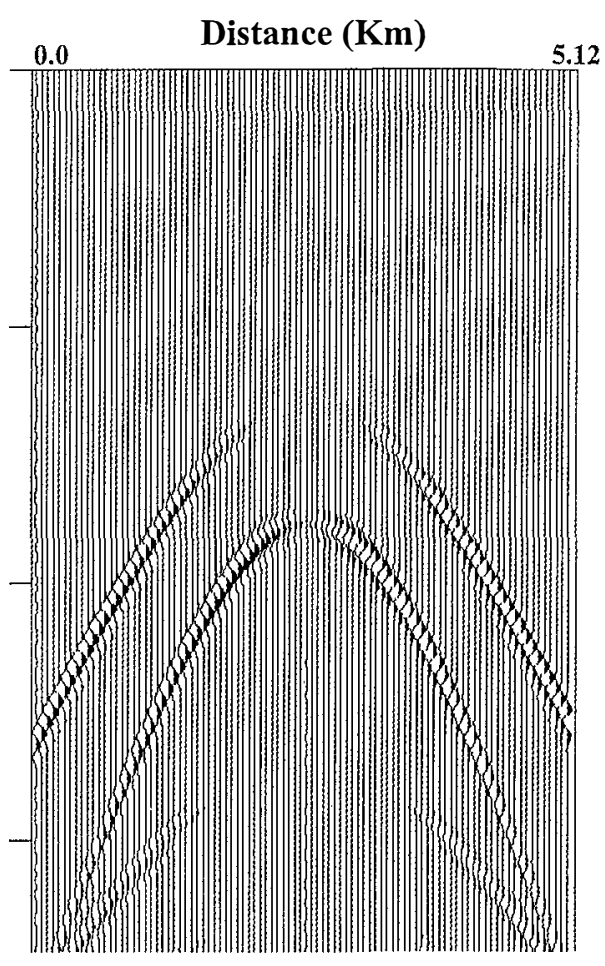

Viscoelastic

Fig. 5. The effects of source type on synthetic seismograms. Under the same source and receiver geometry, the corresponding horizontal component of synthetic displacement seismograms is obtained from a simultaneously excited pressure and shear source in the models of Fig. 1. The seismic responses become complicate compare to the pure pressure source shown in Fig. 4.

raised out of economic and agricultural concerns. To be sure, numerical modeling serves as a basic tool in understanding the fundamental properties that are being investigated in seismic records. Thus, it is important to illustrate some of the basic features in viscoelastic modeling along with its potential capability and the parameters required for its practical usage. Storing oil and gas reserves in old reservoirs has long been a common practice, but the effects of fracture distribution and the corresponding time-lapsed monitoring of oil/gas leakage can very effectively be evaluated in advance through numerical studies by examining seismic responses.

The model, shown in Fig. 6, is composed of 3 layers with an evenly distributed fracture zone. To see detailed wave propagation resulting from the effects of a fractured anticline structure, the layout of the receiver array is located in the second layer, directly over the anticline. Such receiver geometry is not uncommon, especially when a horizontal drill hole is deployed in the vicinity of a source rock during the extraction and injection stages. For the numerical calculations, a grid size of $0.02 \mathrm{~km}$, a time sampling of 0.001 seconds and a source dominant 


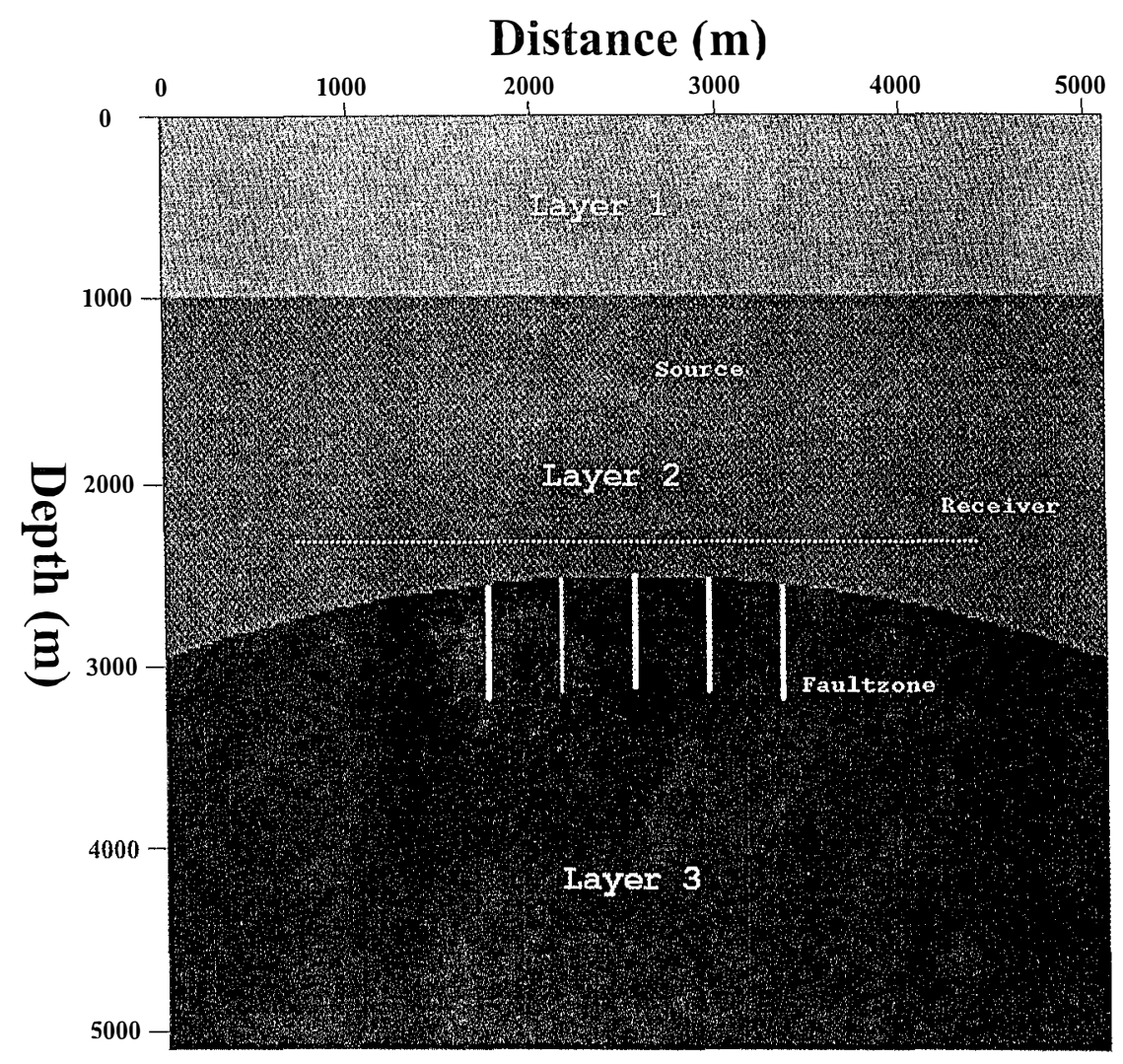

Fig. 6. Model of three layers and a fracture/fault zone in anticline structure of Layer 3. Evaluation of their seismic responses for a specified source and receiver configuration is an important issue for evaluating potential reservoir environment or for investigating the scattering and amplitude variation effects associated with fracture system.

Table 1. Parameters for the model of three layers with fracture zone.

\begin{tabular}{|l|c|c|c|c|}
\hline & Vp $(\mathrm{m} / \mathrm{s})$ & Vs $(\mathrm{m} / \mathrm{s})$ & Qp & Qs \\
\cline { 2 - 5 } & 4400 & 2500 & 100 & 60 \\
\hline Layer 1 & 5000 & 3000 & 200 & 100 \\
\hline Layer 1 & 6000 & 3500 & 1000 & 500 \\
\hline Fractured Z & 3000 & 1700 & 100 & 65 \\
\hline
\end{tabular}

frequency of $50 \mathrm{~Hz}$ are used, a design as in Example 1. The parameters of the P-and S-wave velocities, as well as the quality factors of each layer in the model are shown in Table 1 . The source position is in the middle $(2,560 \mathrm{~m})$ and at the depth of $1,500 \mathrm{~m}$ in a vertical open hole. 
(a) Trace Number
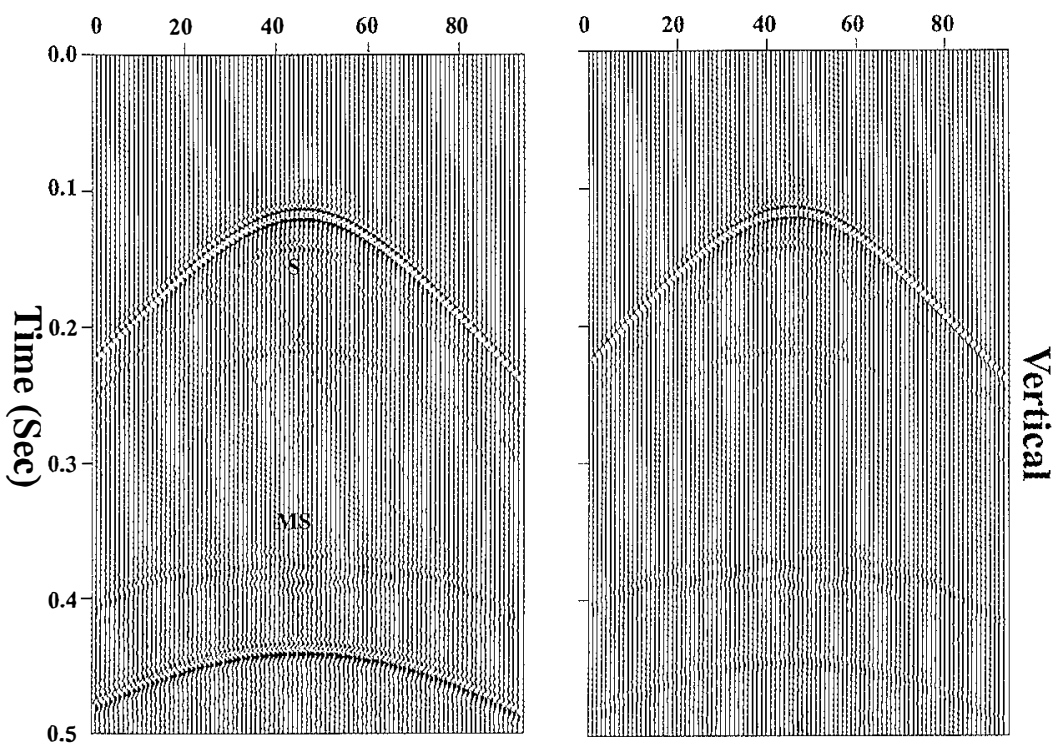

(b)

Trace Number
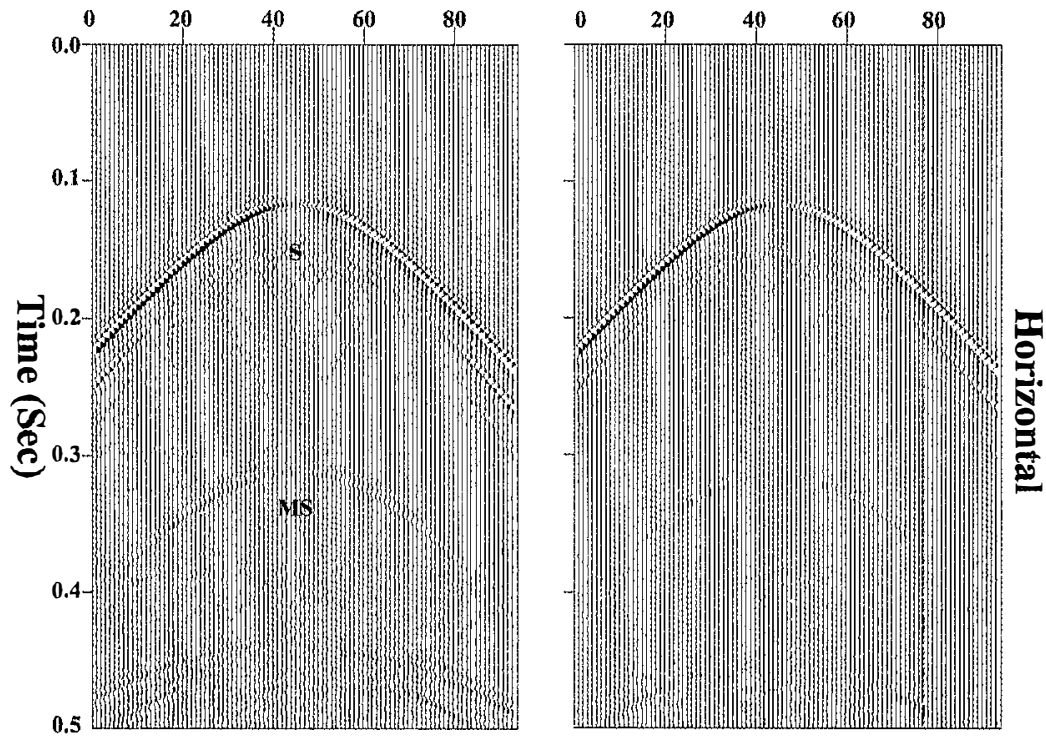

Fig. 7. (a) Synthetic seismograms of vertical component in elastic (left) and viscoelastic (right) seismic wave simulation. Scattering (S) and multiply scattered (MS) waves from fracture zone are discernable in both approach. Comparing the relatively strong free-surface reflection immediately after MS, viscoelastic responses predict more realistic phenomena than elastic approach. (b) Synthetic seismograms of horizontal component in elastic (left) and viscoelastic (right) seismic wave simulation. Multiply scattered (MS) waves are less visible compare to the vertical component show in Fig. 7a. 
The total number of receivers is 93 and the offset distance between them is $40 \mathrm{~m}$, with the first located at $800 \mathrm{~m}$ horizontally and at 2,400 $\mathrm{m}$ in depth. To simulate wave propagation within the seismic prospect area, only a pressure source is generated.

The general features of the attenuation effects in elastic and viscoelastic media with and without explicit consideration of quality factors $(\mathrm{Q})$ are implemented to compare their corresponding responses. The synthetic horizontal and vertical component seismograms are shown in Figs. 7a, b, respectively. It is apparent that transmission, reflections and P-to-S wave conversions at the boundaries of the layers are well depicted in the calculated synthetic responses. In addition, diffractions associated with the primary and multiple reflected P-waves from fractures in the hinge area of the anticline structure in layer 3 are easily identified. Such scattered waves are important in determining the existence of the fracture and the corresponding density distributions within the area of investigation. By observing the synthetic records in Fig. 7, it is evident that the amplitudes for both the P- and S- waves in the viscoelastic medium are much more highly attenuated than those in the elastic media. If the receiver array is located on the surface, the seismic response may be contaminated by near-surface effects, including scattering waves generated by the topography, multiples generated within weather layers and culture noises. Therefore, to evaluate the problem of potential leakage within a highly fractured oil/ gas reservoir environment, it is suggested that viscoelastic responses should be considered under the source and receiver geometry proposed here.

\section{DISCUSSION AND SYNOPSIS}

This study provides the theoretical background and synthetic examples for the effects of viscoelasticity in synthetic fixed-time snapshots and in seismograms computed for models that represent various geological conditions for which realistic viscoelastic parameters may be estimated. The main characteristics associated with wave propagation in viscoelastic media are demonstrated and compared with the corresponding elastic responses through the implementation of viscoelastic theory. Spatial variations in Q values are initially expected to correlate with velocities and density under most geologic conditions. It is found that the coupling of the velocity and the attenuation anomalies associated with structure boundaries and their changes may be decomposed and individually determined for a more detailed investigation. Such an approach, however, may not be necessary at all, unless, of course, a preliminary field data study indicates otherwise. Reflectivity becomes a complicated function of the interaction of all of the variables Vp, Vs, Qp, and Qs, so the interpretation of seismograms in terms of pure elastic or acoustic variables may not be reliable (Kang and McMechan 1993). In a study involving seismic wave propagation modeling, the present implementation of viscoelastic theory can, without a doubt, be used to estimate the parameters of the given medium.

This study also presents and discusses the intrinsic viscoelastic properties which reduce amplitudes and which lead to attenuation and dispersion in their corresponding seismic responses. Scattering effects from fractured media can be simulated through model construction by introducing the effects from discontinuous structure or undulation of structure boundary in our current approach. The secondary scattering effects, such as conglomerate formation 
or debris flow exist in the near-surface for the study of wave propagation phenomena are not explicitly included, they could certainly be directly included by introducing appropriate spatial perturbations in the numerical model parameters, and this will be the topic of our next studies. More specifically, an extension of the current algorithm to include anisotropy and multiple scattering will be the central themes of our future research.

Acknowledgments This research is mainly supported under the grant number of 00-6109 by the agent of Ministry of Science and Technology in Korea. Seismic source signature simulation and the corresponding computation on a Linux PC-cluster were introduced and performed through the international cooperative research with the second author. This paper is also partially supported under grant number of NSC 91-2119-M-194-005 by the second author. The manuscript benefits from comments by Bor-Shouh Huang and very constructive review by $\mathrm{T}$. K. Wang.

\section{REFERENCES}

Aki, K., and B. Chouet, 1975: Origin of coda waves: Source, attenuation and, scattering effects. J. Geophys. Res., 80, 3322-3342.

Alterman, Z. S., and F. C., Karal, 1968: Propagation of elastic waves in layered media by finite-difference methods. Bull. Seism. Soc. Am., 58, 367-398.

Anderson, J. G., and S. Hough, 1984: A model for the shape of the Fourier amplitude spectrum of acceleration at high frequencies. Bull. Seism. Soc. Am., 74, 1969-1993.

Carcione, J. M., D. Kosloff, and R. Kosloff, 1988a: Wave propagation in a linear viscoacoustic medium. Geophys. J., 93, 393-407.

Carcione, J. M., D. Kosloff, and R. Kosloff, 1988b: Wave propagation in a linear viscoelastic medium. Geophys. J., 95, 597-611.

Carcione, J. M., D. Kosloff, and R. Kosloff, 1988c: Viscoacoustic wave propagation simulation in the Earth. Geophysics, 53, 769-777.

Carcione, J. M., 1990: Wave propagation in anisotropic linear viscoelastic media: theory and simulated wavefields. Geophys. J. Int., 101, 739-750.

Chen, H. W., and G. A. McMechan, 1992a, Effects of source configuration on seismograms. Journal of Seismic Exploration, 1, 39-48.

Chen, H. W., and G. A. McMechan, 1992b: Computation of multi-attribute seismic wavefields by solution of the elastodynamic equations. Bull. Seism. Soc. Am., 82, 1134-1143.

Chen, H. W., and G. A. McMechan, 1992c: 3-D pre-stack depth migration for salt and subsalt structures using reverse-VSP data, Journal of Seismic Exploration, 1, 281-292.

Chen, H. W. and G. A. McMechan, 1993: 3-D physical modeling and pseudospectral simulation of seismic common-source data volumes, Geophysics, 58, 121-133.

Chen, H. W., 1996: Staggered-grid pseudospectral viscoacoustic wave field simulation in two-dimensional media. Journal of Acoust. Soc. of Am., 100, No. 1, 120-131.

Clouser, R. H., and C. A. Langston, 1991: $Q_{B}-Q_{s}$ relations in a sedimentary basin using converted waves. Bull. Seism. Soc. Am., 81, 733-750. 
Dougherty, M. E., and R. A. Stephen, 1988: Seismic energy partitioning and scattering in laterally heterogeneous ocean crust. Pure Appl. Geophys., 128, 195-227.

Fehler, M., M. Hoshiba, H. Sato, and K. Obara, 1992: Separation of scattering and intrinsic attenuation for the Kanto-Tokai region, Japan, using measurements of S-wave energy versus hypocentral distance. Geophys. J. Int., 108, 787-800.

Fornberg, B., 1987: The pseudospectral method: comparisons with finite differences for the elastic wave equation. Geophysics, 52, 483-501.

Frankel, A., and L. Wennerberg, 1987: Energy-flux model of seismic coda: Separation of scattering and intrinsic attenuation. Bull. Seismo. Soc. Am., 77, 1223-1251.

Grant, F. S., and G. F. West, 1965: Interpretation theory in applied geophysics: McGraw-Hill, New York, 581 pp.

Gazdag, J., 1973: Numerical convective schemes based on accurate computation of space derivatives. J. Comp. Phys., 13, 1175-1183.

Holberg, O., 1987: Computational aspects of the choice of operator and sampling interval of numerical differentiation in large-scale simulation of wave phenomena. Geophys. Prosp. 35, 629-655.

Kang, I. B., and G. A. McMechan, 1990: Two-dimensional elastic pseudo-spectral modeling of wide-aperture seismic array data with application to the Wichita Uplift- Anadarko Basin region of Southwestern Oklahoma. Bull. Seism. Soc. Am., 80, 1677-1695.

Kang, I. B., and G. A. McMechan, 1993: Viscoelastic seismic responses of 2-D reservoir models. Geophys. Prospect., 41, 149-163.

Kosloff, D., and E. Baysal, 1982: Forward modeling by a Fourier method. Geophysics, 47, 1402-1421.

Kosloff, D., M. Reshef, and D. Lowenthal, 1984: Elastic wave calculation by the Fourier method. Bull. Seism. Soc. Am., 74, 875-891.

Kreiss, H. O., and J. Oliger, 1972: Comparison of accurate methods for the integration of hyperbolic equations. Tellus, 24, 199-255.

Mayeda, K., S. Koyanagi, M. Hoshiba, K. Aki, and Y. Zeng, 1992: A comparative study of scattering, intrinsic, and coda $\mathrm{Q}^{-1}$ for Hawaii, Long Valley, and central California between 1.5 and 15.0 Hz. J. Geophys. Res., 97, 6643-6659.

Olsen, K. B., and G. T. Schuster, 1995: Causes of low-frequency ground motion amplification in the Salt Lake Basin: The case of the vertically-incident $\mathrm{P}$ wave. Geophys. J. Int., 122, 1045-1061.

Orzag, S. A., 1981: Spectral methods for problems in complex geometries. J. Comp. Phys., 37, 70-92.

Phillips, W. S., W. H. K. Lee, and J. T. Newberry, 1988: Spatial variation of crustal coda Q in California. Pure Appl. Geophys., 128, 251-260.

Reshef, M., D. Kosloff, M. Edwards, and C. Hsiung, 1988: Three-dimensional elastic modeling by the Fourier method. Geophysics, 53, 1184-1193.

Toksöz, M. N., A. M. Dainty, E. Reiter, and R.-S. Wu, 1988: A model for attenuation and scattering in the earth's crust. Pure and Appl. Geophys., 128, 81-100. 